\title{
An Improved Fuzzy Logic Based Recommender System by Integrating Social Tags and Social Networks Information
}

\author{
Asgarali Bouyer \\ Faculty of Computer Engineering and Information Technology \\ Azarbaijan Shahid Madani University \\ Tabriz, Iran \\ a.bouyer@azaruniv.edu
}

\begin{abstract}
With the rapid development of computers, internet, social media and networks, and other digital multimedia technologies, it is needed to use a mechanism that can predict the needs and desires of users and recommend the bests for them. Introducing the social networks' information into the traditional collaborative filtering (CF) algorithm, the essay studies the changes of user preference in social networks. Recently a lot of research efforts have been spent on building recommender systems by utilizing the abundant online social network's data. This paper proposes an improved collaborative filtering algorithm based on fuzzy logic and Social Network Information. The proposed method enhances the accuracy of recommendations by combining the social tags, fuzzy logic and social networks' information such as friendship and groups' membership. Through the experiment, the improved algorithm has higher accuracy than the traditional filtering algorithms in the top- $N$ recommendation list. It proves that the social networks' information of users can affect the user's preference.
\end{abstract}

Keywords: Fuzzy Logic, Recommender System, Collaborative Filtering, Social Tags, Social Network

Received: 1 May 2018, Revised 3 June 2018, Accepted 12 July 2018

DOI: $10.6025 / \mathrm{pca} / 2018 / 7 / 2 / 47-55$

(C) 2018 DLINE. All Rights Reserved

\section{Introduction}

Recommender systems have attained widespread acceptance and have attracted the increased attention by the masses for over a decade. Recommender systems can be defined as programs which attempt to recommend the most suitable items (products or services) to particular users (individuals or businesses) by predicting a user's interest in an item based on related information about the items, the users and the interactions between items and users[1,2]. Therefore one of the fundamental aims of these systems is gathering information related to user preferences and items in the system such as movies kinds, and so on [3]. There are variety of resources and methods to collect such information. With the rapid development of internet in recent years, the Internet has transformed from a static platform for propagating and sharing information to a social interaction platform with greater participation and communication. Many famous online social networks, such as Facebook, Myspace, Google+ and Twitter, have recently been emerged. Since the dynamic and open web service environment is quite similar to the social network environment, researchers can use social network information to improve the quality of recommendations. The purpose of the various algorithms fulfills the needs such as precision, innovation and sustainability of the recommended algorithms. Social networks provide a flexible environment which users can interact with other users [4]. 
Recommender systems can be divided into three general categories: Collaborative Filtering (CF) methods, the Content-based methods and the combination of content and collaborative filtering methods. Currently CF methods is mainly divided into two categories: User-based Collaborative Filtering, and Item-based Collaborative Filtering [5]. The User-based Collaborative Filtering algorithm is the oldest algorithms in recommender systems. Goldberg has applied User-based Collaborative Filtering algorithm into email filtering system [6], and Grouplens has then applied the User-based Collaborative Filtering algorithm into news filtering [7]. But the key shortage of User-based Collaborative Filtering algorithm is data sparseness; the diversity of recommend list and the scalability of recommend system. The content based methods, offer the characteristic of the items which has the highest popularity among the known peoples such as directors, actors and others. The combination methods hybrid two methods to use the advantages of both them.

Since the similarity between products/services or between users is naturally uncertain, fuzzy set theory lends itself well to handling the fuzziness and uncertain issues in recommendation problems [8]. More importantly, fuzzy set techniques can be applied to tackle linguistic variables, which are used in describing customer preference, and have the ability to support recommendation generation using uncertain information. In this paper, we have proposed a new approach to enhance recommendation's effectiveness by combining social network's information and CF methods with using fuzzy logic. First, we collected data about users' preference ratings and their social network relationships from a social networking Web sites. Next, we have developed approaches for selecting neighbors and amplifying friends' data. Finally, we generate recommendations about items using CF and the suggested neighbor groups, and compared the performances of diverse algorithms in terms of precision, recall and RMSE metrics.

The rest of this paper is organized as follow. Section 2 discusses the recent studies in CF-based recommender systems and also highlights the associated challenges. In section 3, the proposed method is introduced and discussed in detail. The obtained results from the proposed method are reported in section 4 and the conclusion and final remarks are given in section 5 .

\section{Related Work}

Collaborative filtering (CF) is one of the most popular recommendation techniques, which is used to filter information, this system predicts user preferences based on the opinions of other similar users who have rated the items according to preference [9]. The CF algorithms can be categorized into three main groups: Memory-based CF, Model-based CF, and Hybrid CF. In order to find K nearest neighbors in memory based CF the similarity between two users or items can be calculated based on Pearson Correlation Coefficient similarity, cosine similarity, or Jaccard similarity [10]. Then, according to the nearest neighbors' opinions the most interesting items for the target user are recommended. Memory based algorithms used user rating matrix and make recommendations based on the items rated by the users in the past times. On the other hand, the model based algorithms learn the model with using the user ratings that subsequently are used to perform the task of prediction [11]. Recommender systems that use a combination of two filtering techniques are called hybrid systems.

With the rapid development Web 2.0 technologies, Web users from different backgrounds began to annotate/tag resources on the Web at an incredible speed. Many social media applications like Flickr, Del.ici.ous, and Last.fm provide features which allow users to assign tags to information on the web, i.e. images, blog entries, or video clips. Collaborative tagging has proven to be intuitive for user to adopt by selecting a previously-used tag; it is easy to retrieve annotated resources [12]. In [13], a group of tags are offered to the user based on several criteria (coverage, popularity, effort, uniformity) resulting in a cluster of a relevant tags. Therefore, by using these tags, the proposed method can recommend according to the past tags witch used by several users. With the intrinsic social property of the Web, a large range of applications and services make the user becoming more interactive with Web resources, and a lot of information that concerns both users and resources is constantly generated. This information can be very useful in information retrieval tasks for both user and resources modeling. However, classical models of IR are blind to this social context that surrounds both users and resources. Extracting the data from social networks is very popular recently; fusing social networking information with recommender systems for increasing the level of personalization has received a significant attention from the research communities [14]. Tag-based systems enriched our description of items while social networking systems opened up the door for developing more accurate graphs arising from users' relationships. An active interest is observed in involving tags in CF in recent literature proposed a tag-based contextual CF by using the overlaps of tags [15]. Liu and Lee [4] proposed new method which enhances the accuracy of recommender system when the social networking information is incorporated to CF algorithm. In this method, users' preference ratings data and their social network relations are collected. Then, the Pearson correlation coefficients are used to calculate the nearest neighbors. Finally, if the social network members are in the list of nearest neighbors the member's preference is amplified.

\begin{tabular}{llllll}
\hline 48 & Progress in Computing Applications & Volume & 7 & Number 2 & September 2018 \\
\hline
\end{tabular}


Social network analysis is an analysis of the relationship of the social activists (node) that can achieve the goal via revealing the overall structure of the network, the structure of subgroup, and the influence and interactive mode between the individual and subgroup in the network [16]. Nowadays, many researchers have researched the social network analysis used in personalized recommendation, and most of them combine social network analysis with other methods such as fuzzy logic to make out the final results [17]. Fusing social networking information with recommender systems for increasing the level of personalization has received a significant attention from the research communities [18]. Friends' recommendations are preferred when we compare recommendations from friends with generated recommendations via collaborative methods. Because friends usually share common tastes and interests and it is easy to find the trusted users by the given user based on her/his friendship relations [19].

Seo et al. [20] proposed a new measure to calculate the closeness between users in a social circle, namely, the friendship strength. Further, they proposed a friendship strength-based personalized recommender system that recommends topics or interests users might have in order to analyze big social data, using Twitter in particular. Park et al. proposed the implicit method to finding influential users which considers behaviors and interactions among users to improve the user similarity computation [21]. Porcel et al. developed a fuzzy linguistic based recommender system combining CB filtering and the multi granular fuzzy linguistic modeling technique, which is useful for assessing different qualitative concepts [22]. The authors in [23] proposed a fuzzy linguistic recommender system that operates in university digital library environment and disseminates the stored information to users working in similar domains. The tool is based on the concept of Google Wave, which provides a common space by permitting the users and resources to work together. The approach deals with the cold start problem for new users. To enter in the system, the new users need to define their profiles by selecting a two-tuple linguistic value. The new user's preferences are represented as a vector that subsequently is compared with the other vectors to determine the similarity.

\section{The Proposed Algorithm}

The proposed personalized recommender algorithm is primarily based on CF. CF-based recommender systems apply people relationships for provide recommendations to users by using the information of their directly connected users or friends which is very useful for improving the recommendation quality. Because the strongly connected users had a greater positive influence on each other than the weakly connected users. Furthermore, Fuzzy logic has been used to pinpoint the boundaries of interest among users, which is used in the selection phase of a friend and membership.

\subsection{Measuring Similarity}

The proposed method consists of two parts: the similarity measuring and making recommendation. In part of similarity measurements between users, who will try with an obvious fuzzy method that could be used to display well the relations between human beings with respect to (basis of factors) friendship , membership in groups and tags.

\subsubsection{Friendship-Based Similarity}

There are different ways for calculating the similarity between the members, but in this paper for the first time a fuzzy method is used. Suppose that $T_{i}$ represents the specified period of time and SimMovie[Ti] $[k]$ shows the number of similar watched movies by users $u$ and $v$ in a specified time for movie $k$. Therefore using the following pseudo code, after calculating the number of common movies of people within the specified time, the friendship similarity is calculated by the Eq.(1):

- For each movie in data set

o For each user $u, v$ Which seen movie $k$

- SimMovie $\left[T_{i}\right][k]++;$ Where date $=\{T 1, T 2, \ldots, T 7\}$

- The friendship similarity is calculated by Fsim as follow:

$$
\text { Fsim }=\left\{\begin{array}{c}
(i+1) \times t_{k} \times \text { SimMovie }\left[T_{i}\right][k] \\
\text { Where } T_{i}=\left\{T_{3, \ldots .,}, T_{7}\right\} \text { for } i=\{3,4, \ldots, 7\} \\
t a_{k} \times \text { SimMovie }\left[T_{i}\right][k] \\
\text { Where } T_{i}=\left\{T_{1}, T_{2}\right\} \text { for } i=\{1,2\}
\end{array}\right.
$$


Where $k$ is the name of the movie that has been viewed by two users at a specified interval time and used the $t a$ tag for that movie.

\subsubsection{Membership-Based Similarity}

In this paper, for the first time, different types of films were used for obtaining users belonging to the same group. Nevertheless, selecting a film cannot be regarded as a suitable criterion for placing the users in that group. Hence, a fuzzy approach was used for creating and placing users in different groups which indicates the user's interest in a particular film. In general, there are different groups for different film genres such as action film, science fiction, criminal, comedy, romance, drama, etc. where there is a label for each film genre: $m_{i}=$ \{action film, science fiction, criminal, comedy, romance, drama, etc. $\}$. With using the below pseudo code, the number of movies that user $u$ have seen from different groups in different time periods is considered as a criterion and degree of belonging to that group.

- For each movie in data set

- For each user $u$ Which seen movie $k$ in $T_{i}$

- If MovieKind $[k]==m_{j}$ and $t a_{k} \geq 3$

- MovieMembership $\left[m_{j}\right][k]\left[T_{i}\right]++$;

Where $j=\{1,2, \ldots, 10\}$

The degree of membership in the mentioned groups is obtained by Eq. (2):

$$
\text { Msim }=\left\{\begin{array}{l}
t_{k} \times \text { MovieMembership }\left[m_{j}\right][k]\left[T_{i}\right] j>3 \\
\text { and MovieMembership }\left[m_{j}\right][k]\left[T_{i}\right]>2 \\
\text { MovieMembership }\left[m_{j}\right][k]\left[T_{i}\right] \\
\text { and MovieMembership }\left[m_{j}\right][k]\left[T_{i}\right]>(5-j)
\end{array}\right.
$$

Which, $T_{i}$ represents the time intervals: $\{\mathrm{T} 1, \mathrm{~T} 2, \ldots, \mathrm{T} 7\}$ And $m j$ are different movie groups, and $k$ represents the name of the movie.

\subsubsection{Tag-based Similarity}

To calculate the similarity between users based on the tags the Eq. (3) is used.

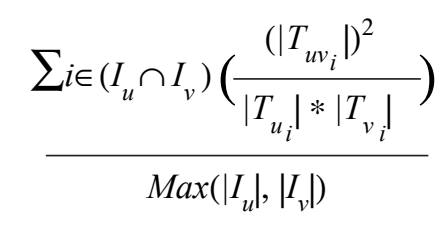

In which, Tuvi set of shared tags between users $u$ and $v$, is for movie $i$, Tui set of tags that have been assigned by the user $u$ for movie $i$ and $I u$ is the u user's movie collection. Max $(|I u|,|I v|)$ indicating the total number of movies, which were selected by the user $u$ and the user $v$.

\subsubsection{The Developed Similarity}

The overall developed similarity between two users $u$ and $v$ is calculated as follow:

$$
\operatorname{Sim}_{u, v}=\alpha * \operatorname{TSim}_{u, v}+(1-\alpha)\left(\beta * \operatorname{FSim}_{u, v}+(1-\beta) * \operatorname{MSim}_{u, v}\right)
$$

Where $\alpha$ and $\beta$ are used in order to make the logical connection between the various criteria of friendship, membership and tags. $\alpha$ was used for maintaining the relation between label, on the one hand, and friendship and membership on the other hand. And, $\beta$ was used for maintaining a relationship between friendships and membership. If $\alpha$ value is high, it denotes the label has higher impact in the similarity of two users. 


\subsection{Making Recommendation}

A subset of users similar to the targeted user was measured based on similarities. Then, the set of their rating was applied for making recommendations to the user. At the next, a user's future behavior is predicted. The output of the recommender system for the targeted user will be a list of their favorite items which he/she would like to do in the future. Measuring individual's interests of user $u$ in a particular item, which has formerly been selected by the neighbor user $v$, depends on two main variables, i.e. the similarity of user $u$ to user $v$ and the similarity of item $i$ which was selected by neighbor $v$ to the items which were formerly labeled by user $u$. For measuring the similarity of items, Jacquard formula (5) was used [24].

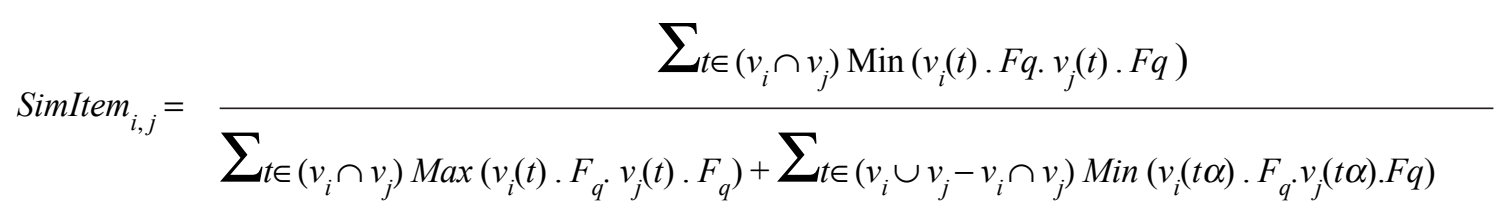

Vector $v_{i}$ for each movie includes the name of label and the number of its repetition. $F_{q}$ denotes the number of repetition of $t$ label on item $i$. The recommendation of a movie to the targeted user is made according to the following algorithm:

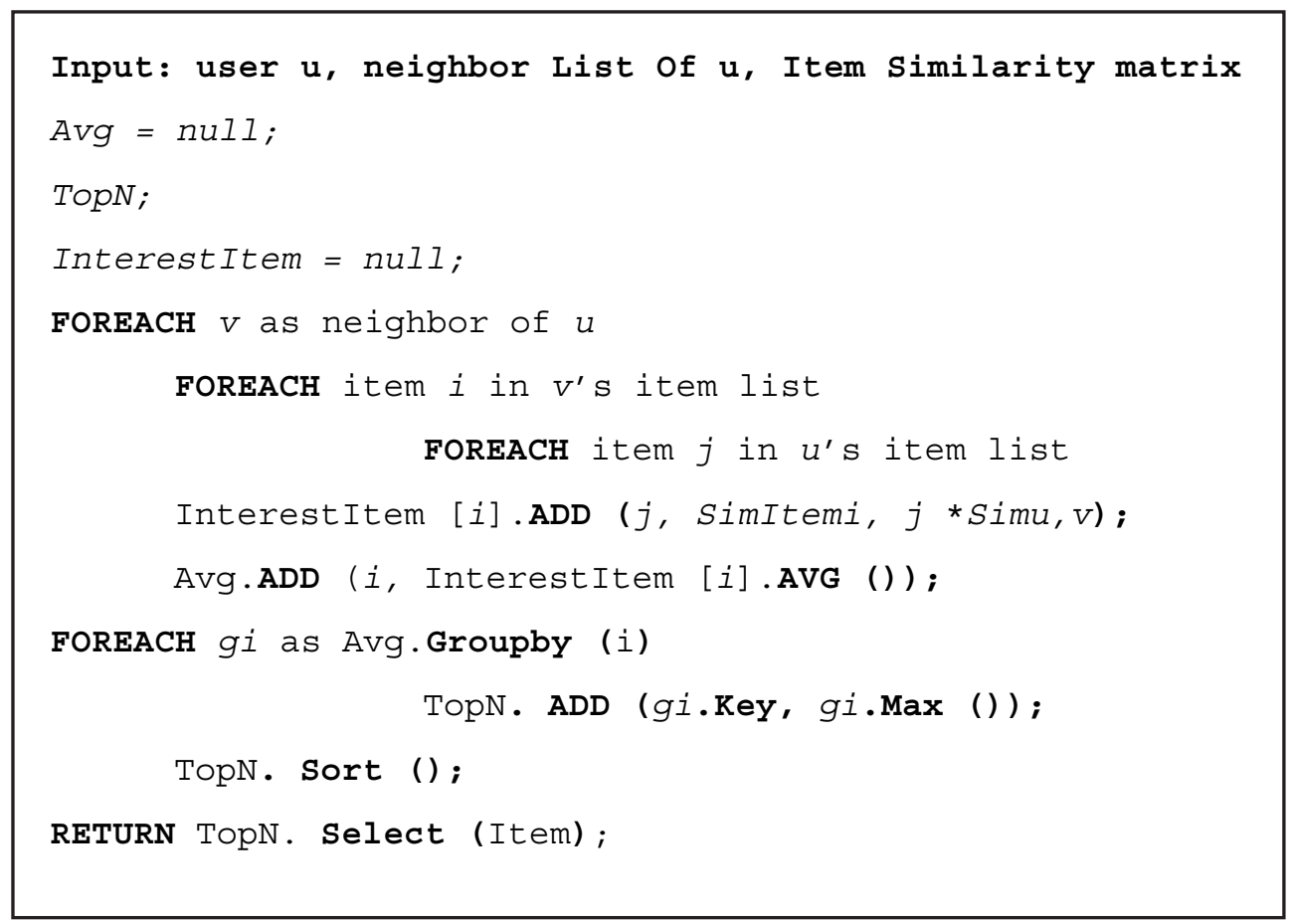

\section{Experimental Results}

\subsection{Data Sets}

The proposed method uses Movie Lens datasets which collected by Group Lens research project at the University of Minnesota. In this work we used Movielens-100K data set of 100,000 Ranking (1-5) from 943 users in 1682 films. Many experiments were performed on the Movie Lens data sets to verify the performance of the proposed method.

We have used the 5-fold cross-validation method for evaluating the precision, recall, and RMSE1 measures for the proposed algorithm and other compared methods. In the 5 -fold cross-validation testing method, dataset is equally divided in 5 different parts. Then algorithm must be executed 5 times and each time one different part ( $20 \%$ of dataset) is considered as test and other four part ( $80 \%$ of the dataset) is selected as the training set. The recommender system recommends a list of ordered items to the test user which has not been selected by this user before. According to the training set information we predict the interest probability of users to those items which are not selected by users. In the test set we have the information of users and their selected items. Thus, in the evaluation part we check for each user if the recommended items based on the training set are the same as the items selected by the user in the test set or not. 


\subsection{Finding $\alpha$ and $\beta$}

$\alpha$ and $\beta$ are used for creating logical and meaningful relations between different criteria of friendship, membership and label. We have used the proposed method in [24] for identifying appropriate $\alpha$ and $\beta$ parameters. As we know, $\alpha$ is used for maintaining relation between labels, friendship and being in the same group. Also, $\beta$ is used for maintaining relation between friendships and membership. For obtaining the most appropriate values for $\alpha$ and $\beta$, it started from zero for $\alpha$ and $\beta$ with a 0.1 hop increase for each value. Then, by conducting experiments and according to the results of the recommendations obtained from Pearson formula, the most appropriate values are obtained. Table 1 shows the obtained result based on Pearson formula for different $\alpha$ and $\beta$. It is clear that the most appropriate values for $\alpha$ and $\beta$ are 0.6 and 0.7 respectively.

\begin{tabular}{|c|c|c|c|c|c|c|c|c|c|}
\hline & \multirow[b]{2}{*}{0.1} & \multicolumn{8}{|c|}{$\alpha$} \\
\hline & & 0.2 & 0.3 & 0.4 & 0.5 & 0.6 & 0.7 & 0.8 & 0.9 \\
\hline 0.1 & 23.49 & 20.12 & 20.11 & 21.78 & 19.11 & 19.87 & 22.67 & 22.64 & 19.34 \\
\hline 0.2 & 21.9 & 18.77 & 20.11 & 21.78 & 19.814 & 21.63 & 24.45 & 22.64 & 18.9 \\
\hline 0.3 & 21.56 & 21.34 & 20.98 & 21.65 & 19.87 & 21.67 & 23.11 & 20.56 & 20.45 \\
\hline 0.4 & 19.78 & 18.74 & 22.11 & 21.23 & 19.67 & 25.07 & 21.97 & 21.11 & 21.78 \\
\hline 0.5 & 21.23 & 19.78 & 22.53 & 19.78 & 18.73 & 17.93 & 18.67 & 19.98 & 19.89 \\
\hline 0.6 & 18.76 & 20.15 & 20.34 & 19.89 & 19.9 & 20.78 & 22.23 & 21.45 & 19.08 \\
\hline 0.7 & 19.94 & 19.86 & 22.34 & 20.75 & 21.21 & 25.67 & 19.87 & 21.43 & 21.32 \\
\hline 0.8 & 20.11 & 18.96 & 19.89 & 19.01 & 18.11 & 20.46 & 21.93 & 21.12 & 20.23 \\
\hline 0.9 & 19.67 & 19.9 & 19.87 & 19.45 & 19 & 20.19 & 21.12 & 20.11 & 21.21 \\
\hline
\end{tabular}

Table 1. Evaluating the Different $\alpha$ and $\beta$ based on Pearson Formula

\subsection{Results}

In this section, we examine the performance of the proposed method with [20] that we call it Seo method. In fact, Seo et al. measure the friendship strength considering communication information between users (interaction similarity), personal similarity and group similarity. They have used different methods such as IP as a combination of interaction and personal similarity, JMSD, and PCC methods. We evaluated the performance of the proposed algorithm and compared it with IP, JMSD and PCC as the baselines. We used PCC for a baseline because it is the most popular with CF-based personalized recommender systems. We also applied JMSD for a baseline because it can consider the ratio of common interest. In addition, JMSD is more suitable for interaction-based recommender systems than other numerical value-based similarity measures.

Our proposed method has been able to produce better results than Seo method due to the presentation of a labeled fuzzy method in finding friends and membership and also with using label. The results of the Precision criterion of the proposed method and the comparative methods are presented in Figure (1).

It should be noted that the results of the proposed method is the average results of at least 50 times repetition of the proposed algorithm in considered datasets. As illustrated by Fig. 1, the proposed method is best suited to the methods being compared. In terms of precision, the proposed method shows an improvement of about $2 \%$ and $1 \%$ as compared to IP for 10 and 20 recommendations, respectively. Our method is also shown to enhance performance by approximately $3.5 \%$ as compared to JMSD and PCC.

The recall criterion shows the correct suggestions with respect to the total suggestions for test datasets. In order to compare the efficiency of the proposed method, the recall criterion is also used to compare the methods in the test data. The results of comparing the proposed method and the comparative methods are presented in Fig. 2, which indicates the superiority of the proposed method. In terms of recall, the proposed method exhibits a performance improvement of about $7.5 \%$ as compared to IP and $11.5 \%$ as compared to PCC and about $13 \%$ as compared to JMSD.

We have also evaluated our method based on RMSEmetric. The RMSE is computed using equation (6). 


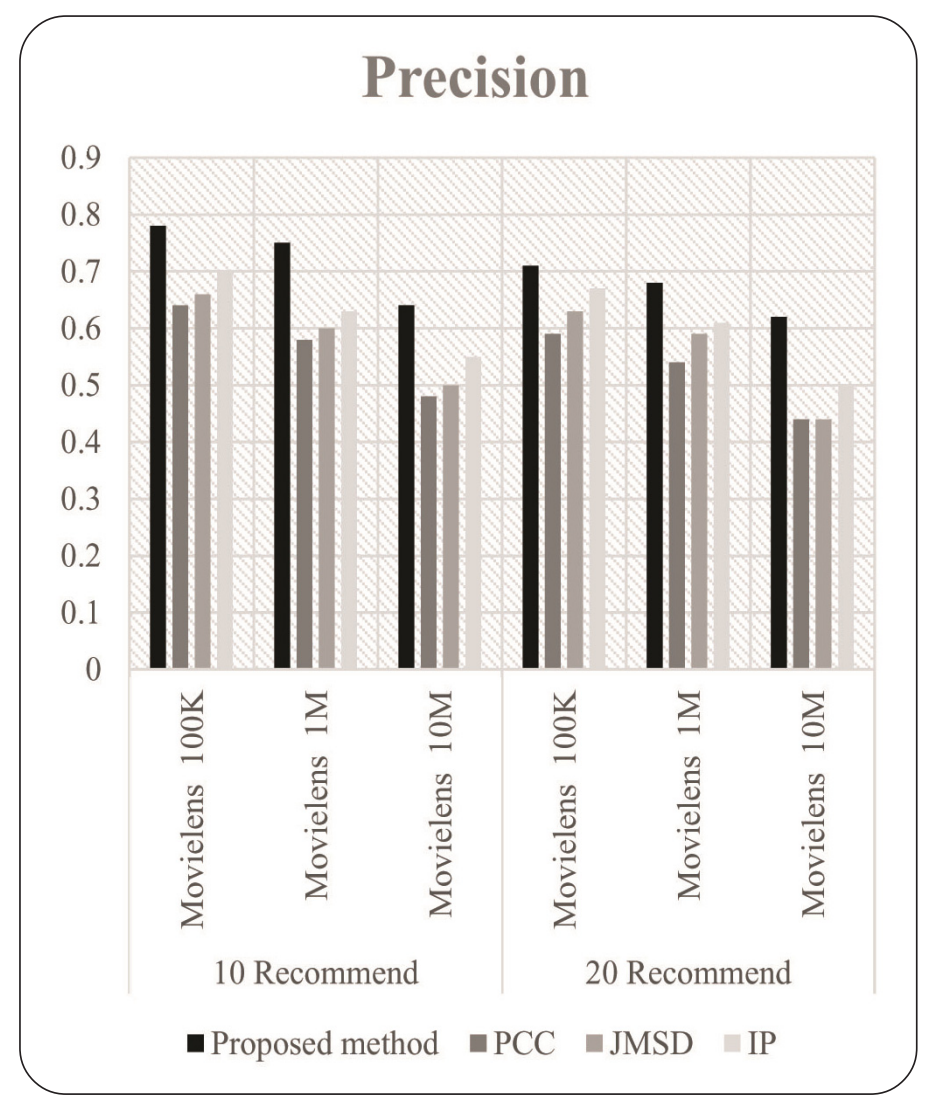

Figure 1. The precision of the proposed method and Seo method [20] with IP, JMSD, and PCC measures

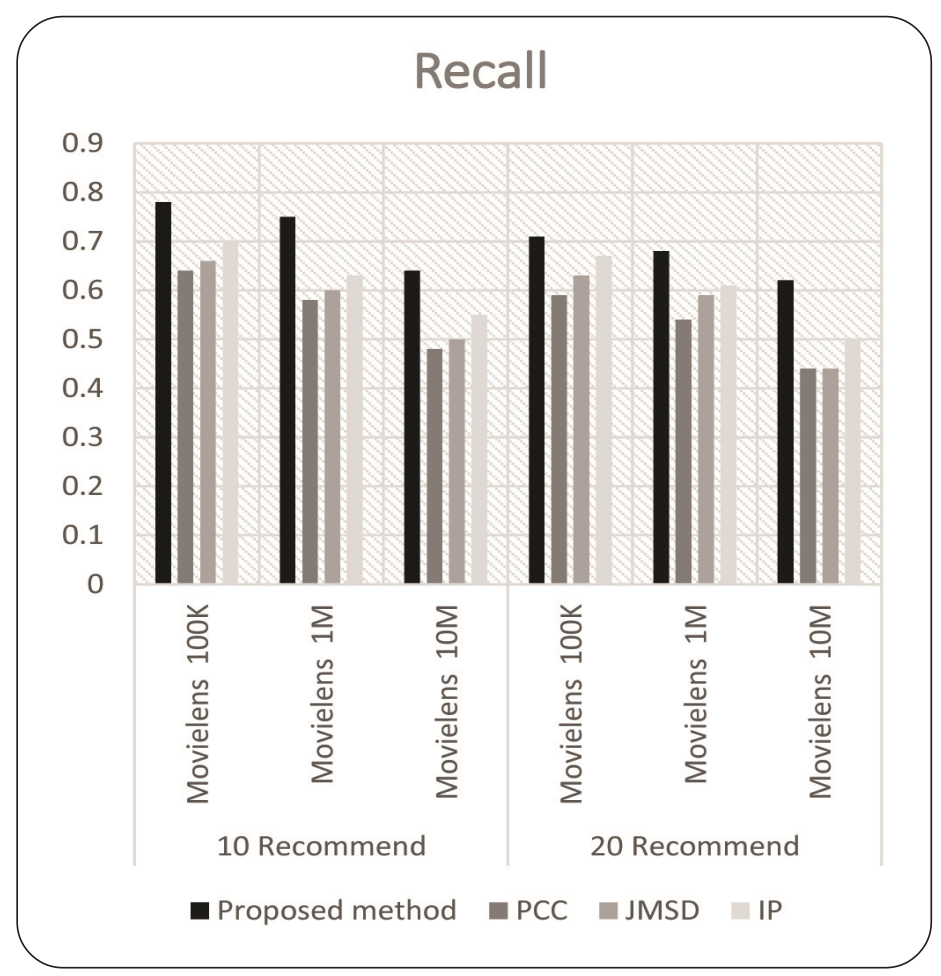

Figure 2. The Recall measure of the proposed method and Seo method [20] with IP, JMSD, and PCC measures 


$$
R M S E=\sqrt{\frac{\sum_{(u, i) \mid R_{u, i}}\left(\hat{r}_{u, i} r_{u, i}\right)^{2}}{N}}
$$

Where $R_{u, i}$ indicates that the user $u$ has submitted a point for the item $i$. The lower the RMSE value, the higher the accuracy of the system's results.

Table 2 shows the obtained RMSE values of the proposed algorithm and other compared methods for five independent tests according to the 5-fold cross-validation. As mentioned before, the lower RMSE value show that the algorithm has lower error for its recommendations. Therefore, it is proved that the combination of the fuzzy algorithm with information of social networks such as friendship, groups' membership and being in similar communities, and considering the time factor significantly improves the recommendation systems in providing accurate suggestions.

\begin{tabular}{|l|l|c|}
\hline \multirow{2}{*}{ Method's name } & \multicolumn{2}{c|}{ RMSE Measure } \\
\cline { 2 - 3 } Proposed Method & 0.9032 & MovieLens 100k \\
\hline BipolarSlopeOne & 0.9432 & 0.905 \\
\hline FactorWiseMatrixFactorization & 0.9263 & 0.9654 \\
\hline BiasedMatrixFactorization & 0.9137 & 0.9208 \\
\hline SVDPlusPlus & 0.9156 & 0.9112 \\
\hline ItemKNNPearson & 0.9141 & 0.9107 \\
\hline
\end{tabular}

Table 2. Results For RMSE Measure on Movielens Datasets based on 5-fold Cross-validation

To evaluate the effectiveness of the proposed method, a comparison between the proposed method and the basic algorithms in this field was carried out. As shown in Table 2, the proposed method has better results than all basic algorithms, which indicates the high accuracy of the proposed method in proposing recommendations to the users. The results show that our proposed method has first rank in RMSE metric and the second rank belongs to SVDPlusPlus methods that has better results than other basic methods. The main factor behind the proposed method is the time factor, which has led to suggestions for films that the neighbor users have observed in time.

\section{Conclusion}

Because of the fast development and growth of internet and e-business it is needed to generate a mechanism which can predict users' needs and requests. Recommender systems were created in line with these developments. As mentioned, recommender systems are characterized as systems which help users in finding and selecting their favorite items. The precision and accuracy of a recommendation is considered to be one of the fundamental challenges of these systems. The previous studies don't consider the behavior changes of users. Due to the capabilities of fuzzy systems in determining the border of users' interests, we used them in this study in combination with social network information. Hence, the combination of social network information such as label, friendship and membership with fuzzy logic was investigated in this paper so as to enhance recommender systems. The results of simulation in C\# program indicated that using social network information based on memory and fuzzy logic leads to the optimization of the accuracy of recommender systems.

\section{References}

[1] Lee, S. K., Cho, Y. H., Kim, S. H. (2010). Collaborative filtering with ordinal scale-based implicit ratings for mobile music recommendations. Information Sciences, 180 (11) 2142-2155.

[2] Choi, K., et al. (2012). A hybrid online-product recommendation system: Combining implicit rating-based collaborative 
filtering and sequential pattern analysis. Electronic Commerce Research and Applications, 11 (4) 309-317.

[3] Wei, S., et al. (2016). A hybrid approach for movie recommendation via tags and ratings. Electronic Commerce Research and Applications, 18, 83-94.

[4] Liu, F., Lee, H. J. (2010). Use of social network information to enhance collaborative filtering performance. Expert systems with applications, 37 (7) 772-4778.

[5] Hong, Y., Zhuanyun, L. (2010). A collaborative filtering recommendation algorithm based on forgetting curve, Journal of Nanjing University (Natural Sciences), 5. 46.

[6] Goldberg, D., et al. (1992). Using collaborative filtering to weave an information tapestry. Communications of the ACM, 35 (12) 61-70.

[7] Resnick, P., et al. (1994). GroupLens: an open architecture for collaborative filtering of netnews. In: Proceedings of the 1994 ACM conference on Computer supported cooperative work. ACM.

[8] Cornelis, C., (2007). One-and-only item recommendation with fuzzy logic techniques. Information Sciences, 177(22) 49064921.

[9] Adomavicius, G., Tuzhilin, A. (2005).Toward the next generation of recommender systems: A survey of the state-of-the-art and possible extensions. IEEE Transactions on Knowledge and Data engineering, 17(6) 734-749.

[10] Nakamoto, R., et al. (2007).Tag-based contextual collaborative filtering. IAENG International Journal of Computer Science, $34(2)$.

[11] Gavalas, D. (2014). Mobile recommender systems in tourism. Journal of Network and Computer Applications, 39, 319-333.

[12] Hammond, T., et al. (2005). Social bookmarking tools (I) a general review. D-lib Magazine, 2 (4).

[13] Xu, Z., et al. (2006). Towards the semantic web: Collaborative tag suggestions. in Collaborative web tagging workshop at WWW2006, Edinburgh, Scotland.

[14] Chiu, Y.-S., K.-H. Lin., Chen, J.-S. (2011).. A social network-based serendipity recommender system. In: Intelligent Intelligent Signal Processing and Communications Systems (ISPACS), 2011 International Symposium on. IEEE.

[15] Michlmayr, E., Cayzer, S. (2007). Learning user profiles from tagging data and leveraging them for personal (ized) information access.

[16] Pérez, L.G., Montes-Berges, B., del Rosario Castillo-Mayen, M. (2011). Boosting social networks in Social Network-Based Recommender System. In: Intelligent Systems Design and Applications (ISDA), $11^{\text {th }}$ International Conference on. 2011. IEEE.

[17] Deng, S., Huang, L., Xu, G. (2014). Social network-based service recommendation with trust enhancement. Expert Systems with Applications, 41 (18) 8075-8084.

[18] Schall, D. (2015). Social network-based recommender systems. Springer.

[19] Ye, M., et al. (2011). Exploiting geographical influence for collaborative point-of-interest recommendation. In: Proceedings of the $34^{\text {th }}$ International ACM SIGIR Conference on Research and Development in Information Retrieval. 2011. ACM.

[20] Seo, Y.-D., et al. (2017). Personalized recommender system based on friendship strength in social network services. Expert Systems with Applications, 69, 135-148.

[21] Park, J., et al. (2010). An online video recommendation framework using view based tag cloud aggregation. IEEE Multimedia, $99(1)$.

[22] Porcel, C., López-Herrera, A.G., Herrera-Viedma, E. (2009).A recommender system for research resources based on fuzzy linguistic modeling. Expert Systems with Applications, 36 (3) 5173-5183.

[23] Serrano-Guerrero, J., et al. (2011). A google wave-based fuzzy recommender system to disseminate information in University Digital Libraries 2.0. Information Sciences, 181 (9) 1503-1516.

[24] Naseri, S., Bahrehmand, A., Ding, C. (2015).An Improved Collaborative Recommendation System by Integration of Social Tagging Data, In: Recommendation and Search in Social Networks.Springer. 119-138. 